\title{
DIAGNÓSTICO DA ARBORIZAÇÃO URBANA EM BAIRROS DO MUNICÍPIO DE PORTO ALEGRE, RS, BRASIL
}

\author{
Bruna de Oliveira Boeni ${ }^{1}$, Denise Silveira ${ }^{2}$ \\ (recebido em 30.01.2011 e aceito para publicação em 15.09.2011)
}

\section{RESUMO}

A arborização proporciona melhorias nas condições ambientais das cidades. As árvores contribuem principalmente na manutenção do microclima, diminuindo a poluição e ainda na conservação da biodiversidade regional. Uma arborização bem planejada poderá garantir a integridade da vegetação em conformidade com a infraestrutura urbana. O objetivo deste trabalho foi diagnosticar a situação da vegetação arbórea nos passeios públicos de nove bairros do município de Porto Alegre, RS, Brasil. Para a amostragem foi utilizado o método qualiquantitativo, sendo identificada a espécie, a ocorrência, o diâmetro à altura do peito, o diâmetro de copa, a altura e o estado fitossanitário. Foram amostrados 4318 indivíduos arbóreos, totalizando 122 espécies, nas quais apenas 45 são nativas e 77 de origem exótica, sendo as mais frequentes: Jacaranda mimosifolia, Handroanthus avellanedae, Lagerstroemia indica e Ligustrum lucidum. Ressalta-se a ocorrência de espécies invasoras como Pinus sp., Hovenia dulcis e Ligustrum lucidum. Quase $80 \%$ dos indivíduos apresentaram bom estado fitossanitário, embora o grande porte destes seja conflitante com a infraestrutura urbana. Portanto, a manutenção e o planejamento das vias públicas devem levar em consideração o plantio de espécies nativas com características ecológicas condizentes com o ambiente urbano, pela substituição de indivíduos exóticos ou comprometidos fitossanitariamente.

\footnotetext{
${ }^{1}$ Bióloga, Mestre em Biologia. Doutoranda no Programa de Pós-Graduação em Botânica, Universidade Federal do Rio Grande do Sul - UFRGS, Departamento de Botânica, Porto Alegre, RS, bruboeni@gmail.com.

${ }^{2}$ Bióloga, graduada no curso de Ciências Biológicas na Universidade do Vale do Rio dos Sinos UNISINOS, São Leopoldo, RS, denisesilvei@gmail.com
} 
Palavras-chave: Arborização urbana; Espécies nativas; Planejamento urbano; Levantamento quali-quantitativo.

\section{URBAN FOREST DIAGNOSIS ON NEIGHBORHOODS IN THE CITY OF PORTO ALEGRE, STATE OF RIO GRANDE DO SUL, BRAZIL}

\section{ABSTRACT}

Urban forests provide improvements in the environmental conditions of cities. Trees contribute mainly for the maintenance of the microclimate, reducing pollution and also helps the conservation of regional biodiversity. A well-planned urban vegetation can ensure the integrity of the vegetation in harmony with the urban infrastructure. The objective of this study was to diagnose the situation of urban trees on public roads on nine neighborhoods in the city of Porto Alegre, Brazil. The sampling, were made based in qualitative-quantitative method, identifying species, origin, diameter at breast-height, crown diameter, height, and phytosanitary state. We sampled 4318 individual plants, totalizing 122 species, 45 of native and 77 of exotic origin. The most common were: Jacaranda mimosifolia; Handroanthus avellanedae; Lagerstroemia indica and Ligustrum lucidum. It is important to highlight the occurrence of invasive species, such as Pinus sp., Hovenia dulcis e Ligustrum lucidum. Almost $80 \%$ of the individuals presented good phytosanitary state, although their large size conflicted with the urban infrastructure. Thus, public road maintenance and planning must take into consideration the planting of native species with ecological characteristics in accordance with the urban environment, through the substitution of exotic individuals or of those in a phytosanitary compromised state.

Keywords: Urban forestry; Native species; Urban planning; Qualitative-quantitative sampling. 


\section{INTRODUÇÃO}

A vegetação urbana é responsável pela melhoria da qualidade do ambiente nas cidades, minimizando efeitos causados pela expansão populacional. A arborização está diretamente relacionada com a qualidade de vida, o aumento da biodiversidade, a preservação das espécies nativas e o bem-estar físico e psíquico do ser humano. As árvores trazem benefícios como fornecimento de sombra e oxigênio, atenuação do calor e contribuição para a estabilidade microclimática, redução da velocidade dos ventos e ruídos, combate à erosão e fornecimento de abrigo e alimento para a fauna (MILANO, 1984; PALERMO, 1985; GODOY, 1995; JACINTO, 2001; MASCARÓ; MASCARÓ, 2002).

Contudo, para que a arborização urbana seja mais eficiente, o planejamento da mesma deve considerar as características das espécies vegetais em relação ao espaço disponível. Este deve ser elaborado de forma que não cause danos aos vegetais e que não entre em conflito com a infraestrutura urbana. Deve considerar também a diversidade das espécies e a ocorrência regional das mesmas, proporcionando o aumento da vida útil da planta (LIMA, 1995). Segundo Velasco et al. (2006), são grandes as dificuldades de se implantar a vegetação nas cidades adaptando a arquitetura natural das espécies arbóreas à presença de instalações hidráulicas, redes elétricas, telefônicas ou sanitárias.

O uso de espécies nativas no planejamento da arborização deve ser priorizado, pois as espécies exóticas podem causar danos ao ambiente, perda da biodiversidade e alteração da paisagem natural (ZILLER, 2001). Entretanto, a maioria das cidades brasileiras apresenta a composição arbórea pouco diversificada, muitas vezes se constituindo de monoculturas e do predomínio de espécies exóticas. Esta homogeneização da vegetação pode contribuir para a perda do equilíbrio ecológico, condição básica à diversidade das espécies (SANTOS; TEIXEIRA, 2001). Segundo Andrade (2002), as espécies usadas na arborização no Sul do Brasil são praticamente as mesmas utilizadas no Centro-Oeste, deixando de se explorar as possibilidades de riqueza das espécies da flora local.

A diversidade, a heterogeneidade e o cálculo da frequência das espécies utilizadas na arborização são de suma importância para evitar os riscos relacionados à fitossanidade, devido ao ataque de pragas e doenças. Isto se faz necessário para garantir o máximo de proteção aos indivíduos vegetais, difundindo e valorizando a flora brasileira e 
consequentemente favorecendo a sobrevivência da fauna nativa (GREY; DENEKE,1978; TOLEDO; PARENTE, 1988; SANTAMOUR-JUNIOR, 2002).

Sendo assim, este trabalho teve o objetivo de realizar um inventário qualiquantitativo na tentativa de avaliar a situação da arborização urbana no que se refere ao uso de espécies nativas e exóticas, o estado fitossanitário e o porte arquitetônico dos vegetais em passeios públicos em nove bairros no município de Porto Alegre, RS.

\section{MATERIAIS E MÉTODOS}

Este estudo foi realizado na zona urbana do município de Porto Alegre, capital do estado do Rio Grande do Sul, Brasil. Segundo a Secretaria Municipal de Meio Ambiente (SMAM, 2002) da cidade, nas ruas são encontradas aproximadamente 173 espécies arbóreas, sendo que aproximadamente $15 \%$ dos indivíduos foram plantados por iniciativa de particulares e o restante oriundos de plantio executados pela Prefeitura. A arborização de vias públicas da capital se iniciou efetivamente na década de 40 (SANCHOTENE, 1990), sendo que atualmente muitas árvores são tombadas como Patrimônio Natural, como o caso dos jacarandás e tipuanas nas ruas do bairro Moinhos de Vento.

A vegetação da região na qual está inserido o município era originalmente coberta por Floresta Estacional Semidecidual em ecótono com as formações vegetais pioneiras, restingas e banhados (TEIXEIRA et al., 1986). A Floresta Estacional Semidecidual é constituída por fanerógamas com gemas foliares deciduais e a perda das folhas do conjunto florestal situa-se entre $20 \%$ e $50 \%$, sendo determinada pelas baixas temperaturas que precedem o inverno, conforme Leite e Klein (1990). Pela classificação de Köppen, o clima está enquadrado na categoria subtropical úmido (Cfa), apresentando temperaturas médias superiores a $22^{\circ} \mathrm{C}$ no mês mais quente, sem estação seca definida durante o ano.

O levantamento de campo foi realizado no período de janeiro de 2008 a novembro 2009, sendo utilizado o método de inventário qualiquantitativo baseado em Filho et al. (2002). Os logradouros e canteiros avaliados foram selecionados para amostragem de acordo com o projeto de instalação de tubulação de gás natural nos bairros: Moinhos de Vento; Jardim Botânico; Humaitá; Boa Vista; Passo da Areia; Rubem Berta; Jardim Lindóia; Vila Jardim e Petrópolis (Figura 1). Assim, possíveis efeitos do empreendimento na arborização pública, como o dano às raízes, podem ser avaliados e monitorados garantindo 
a integridade da vegetação. Para a localização dos indivíduos arbóreos nas vias públicas foi utilizada planta planialtimétrica na escala 1:10.000 com informações da malha viária dos bairros.

No levantamento foram considerados somente os espécimes lenhosos com altura igual ou superior a dois metros. Para todos os espécimes foram apreciados os seguintes aspectos: nome científico; nome popular; família botânica; ocorrência natural (nativa ou exótica à flora original do Rio Grande do Sul); estado fitossanitário, avaliado conforme três categorias estabelecidas: bom, ruim e regular (adaptado de Faria; et al., 2007), (Tabela 1); circunferência à altura do peito ( $1,30 \mathrm{~m}$ do solo), utilizando fita métrica comum (os dados foram transformados para DAP - diâmetro à altura do peito); diâmetro de abertura da copa (DAC) (sentido longitudinal e transversal ao meio fio, sendo aqueles de valor zero relacionados com podas danosas - em sua maioria - e tratamentos fitossanitários emergenciais); altura $(\mathrm{H})$, feita com trena para os indivíduos de porte médio, até 6 metros e estimadas as árvores de grande porte; além da freqüência (\%), calculada através da razão entre número de indivíduos da espécie e o número total de indivíduos. Os dados coletados foram inseridos em um banco de dados construído no software Microsoft Office Excel 2007.

A identificação das espécies botânicas foi realizada através de estruturas vegetativas e reprodutivas, quando necessária foi acompanhada a fenologia do indivíduo. Os exemplares coletados foram identificados através de literatura específica (LORENZI, 2002a; LORENZI, 2002b; LORENZI et al., 2003; BACKES; IRGANG, 2004; SOBRAL et al., 2006; SOUZA; LORENZI, 2008) e o sistema de taxonomia botânica utilizado para as famílias foi o APG III (APG, 2009). 


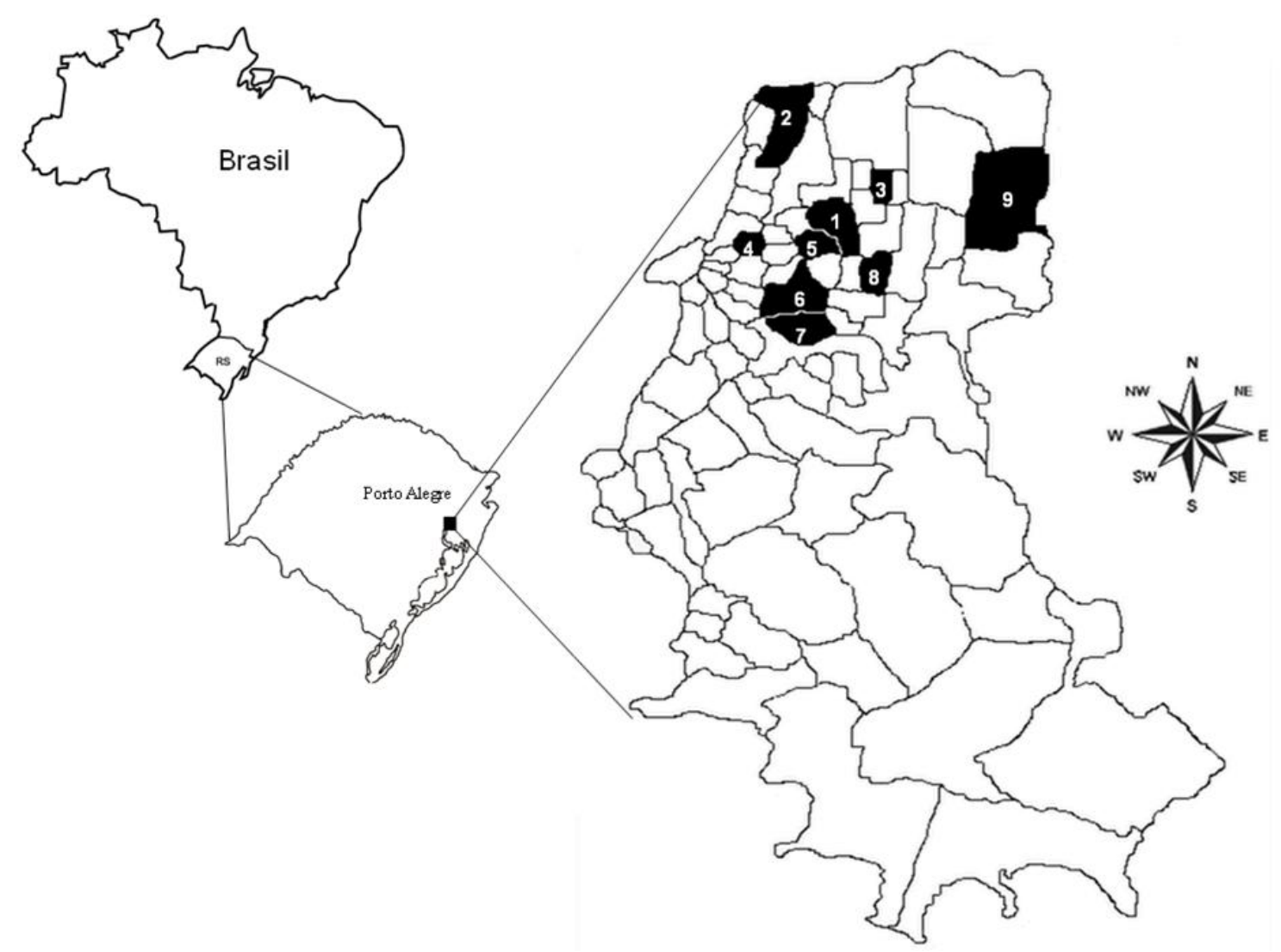

Figura 1. Localização dos limites entre os bairros amostrados no município de Porto Alegre, Rio Grande do Sul, Brasil. Os bairros seguem a respectiva numeração: 1 Passo da Areia; 2 - Humaitá; 3 - Jardim Lindóia; 4 - Moinhos de Vento; 5 Boa Vista; 6 - Petrópolis; 7 - Jardim Botânico; 8 - Vila Jardim e 9 - Rubem Berta

Figure 1. Localization of the neighborhood limits in the city of Porto Alegre, Rio Grande do Sul, Brazil. The neighborhoods follow the respective numeration: 1 - Passo da Areia; 2 - Humaitá; 3 - Jardim Lindóia; 4 - Moinhos de Vento; 5 - Boa Vista; 6 Petrópolis; 7 - Jardim Botânico; 8 - Vila Jardim and 9 - Rubem Berta 
Tabela 1. Critérios utilizados para avaliar as categorias de estado fitossanitário das árvores amostradas (adaptado de Faria et al., 2007)

Table 1. Criteria used to evaluate the categories of phytosanitary state of the sampled trees (adapted from Faria et al., 2007)

\begin{tabular}{|c|l|}
\hline CATEGORIA & \multicolumn{1}{c|}{ CRITÉRIO } \\
\hline Boa & $\begin{array}{l}\text { Árvore vigorosa e sadia, sem sinais aparentes de ataque de insetos, } \\
\text { doenças (tecidos necrosados, secreções caulinares, galhos mortos); não } \\
\text { apresenta infestação acentuada de hemiparasitas (erva-de-passarinho) ou } \\
\text { injúrias mecânicas; pequena ou nenhuma necessidade de manutenção; } \\
\text { forma ou arquitetura característica da espécie. }\end{array}$ \\
\hline Regular & $\begin{array}{l}\text { Médias condições de vigor e saúde, necessitando de pequenos reparos } \\
\text { ou poda, apresentando descaracterização da forma, sinais de ataque de } \\
\text { insetos, doença e sensível ataque por hemiparasitas. }\end{array}$ \\
\hline Ruim & $\begin{array}{l}\text { Avançado e irreversível declínio, apresentando ataque muito severo por } \\
\text { insetos ou injúria mecânica, descaracterizando sua arquitetura ou } \\
\text { desequilibrando o vegetal; lenho corroído ou sinais de doença que } \\
\text { aparentemente comprometem sua existência ou que se apresenta } \\
\text { infestada por hemiparasitas. Para ser recuperada necessita de tratamento } \\
\text { fitossanitário rigoroso. }\end{array}$ \\
\hline
\end{tabular}

Adaptado de Faria et al. ,2007.

\section{RESULTADOS E DISCUSSÃO}

Foram amostrados 4318 indivíduos arbóreos nos passeios públicos avaliados. As espécies inventariadas estão agrupadas em 122 espécies, 94 gêneros e 42 famílias, sendo Fabaceae a mais representativa com $54,8 \%$ do total de espécies, seguida por Myrtaceae (33,3\%), Arecaceae e Moraceae ambas com 16,7\%, além de Malvaceae $(14,3 \%)$ e Bignoniaceae (11,9\%). As demais famílias possuem representatividade abaixo de $10 \%$.

Jacaranda mimosifolia, Handroanthus avellanedae, Lagerstroemia indica e Ligustrum lucidum são responsáveis por $50,9 \%$ do total dos espécimes inventariados (Tabela 2). De acordo com as recomendações para arborização de Milano e Dalcin (2000), cada espécie não deve ultrapassar $10-15 \%$ do total de indivíduos da população para um bom planejamento. No entanto, a espécie de maior frequência, Jacaranda mimosifolia, representa $17,4 \%$ do total. Cabe ressaltar que todas as espécies amostradas com frequência são exóticas à flora original do Rio Grande do Sul. Segundo Santamour-Júnior (2002), a 
diversidade de espécies é necessária, pois evita o ataque de pragas e doenças, levando à deterioração fitossanitária. Da mesma forma, Santamour-Júnior (2002) recomenda não exceder mais que 10\% de indivíduos da mesma espécie e 30\% de uma família botânica.

Tabela 2. Levantamento florístico contendo nome científico e popular, família, ocorrência (N nativa; $E$ - exótica) e valores de frequência absoluta (FA) e relativa (FR) da arborização dos bairros amostrados do município de Porto Alegre, RS, Brasil

Table 2. Forest survey containing scientific and popular name, family, origin ( $\mathrm{N}$ - native; $\mathrm{E}$ exotic) and values of absolute (AF) and relative (RF) frequency of urban forest in the analyzed neighborhoods of the city of Porto Alegre, Rio Grande do Sul, Brazil

\begin{tabular}{|c|c|c|c|c|c|}
\hline Nome científico & Nome popular & Família & Ocorrência & $\mathrm{FA}$ & $\begin{array}{l}\text { FR } \\
(\%)\end{array}$ \\
\hline Jacaranda mimosifolia D. Don & jacarandá & Bignoniaceae & $E$ & 753 & 17,44 \\
\hline $\begin{array}{l}\text { Handroanthus avellanedae (Lorentz ex } \\
\text { Griseb.) Mattos }\end{array}$ & ipê-roxo & Bignoniaceae & $E$ & 518 & 12,00 \\
\hline Lagerstroemia indica L. & extremosa & Lythraceae & $E$ & 516 & 11,95 \\
\hline Ligustrum lucidum W.T. Aiton & ligustro & Oleaceae & $E$ & 409 & 9,47 \\
\hline Tipuana tipu (Benth.) Kuntze & tipuana & Fabaceae & $E$ & 223 & 5,16 \\
\hline $\begin{array}{l}\text { Handroanthus chrysotrichus (Mart. ex A. } \\
\text { DC.) Mattos }\end{array}$ & ipê-âmarelo & Bignoniaceae & $E$ & 209 & 4,84 \\
\hline $\begin{array}{l}\text { Brachychiton populneus (Schott \& Endl.) } \\
\text { R. Br. }\end{array}$ & perna-de-moça & Malvaceae & $E$ & 202 & 4,68 \\
\hline Caesalpinia peltophoroides Benth. & sibipiruna & Fabaceae & $E$ & 123 & 2,85 \\
\hline Melia azedarach L. & cinamomo & Meliaceae & $E$ & 107 & 2,48 \\
\hline Syagrus romanzoffiana (Cham.) Glassman & jerivá & Arecaceae & $\mathrm{N}$ & 98 & 2,27 \\
\hline Peltophorum dubium (Spreng.) Taub. & canafístula & Fabaceae & $\mathrm{N}$ & 87 & 2,01 \\
\hline Ficus benjamina L. & figueira-de-jardim & Moraceae & $E$ & 79 & 1,83 \\
\hline Yucca elephantipes Regel & iuca & Asparagaceae & $E$ & 61 & 1,41 \\
\hline Eugenia uniflora L. & pitanga & Myrtaceae & $\mathrm{N}$ & 52 & 1,20 \\
\hline Schizolobium parahyba (Vell.) S.F. Blake & guapuruvu & Fabaceae & $E$ & 48 & 1,11 \\
\hline Parapiptadenia rigida (Benth.) Brenan & angico-vermelho & Fabaceae & $\mathrm{N}$ & 38 & 0,88 \\
\hline Platanus acerifolia (Aiton) Willd. & plátano & Platanaceae & $E$ & 28 & 0,65 \\
\hline Psidium guajava L. & goiabeira & Myrtaceae & $E$ & 28 & 0,65 \\
\hline Persea americana Mill. & abacate & Lauraceae & $E$ & 27 & 0,63 \\
\hline Psidium cattleianum Sabine & araçá & Myrtaceae & $\mathrm{N}$ & 27 & 0,63 \\
\hline Dracaena sp. Vand. ex L. & dracena & Asparagaceae & $E$ & 26 & 0,60 \\
\hline $\begin{array}{l}\text { Allophyllus edulis (A.St.Hil. } \\
\text { A.Juss.\&Cam.)Hie.exNie. }\end{array}$ & chal-chal & Sapindaceae & $\mathrm{N}$ & 25 & 0,58 \\
\hline Caesalpinia ferrea Mart. & pau-ferro & Fabaceae & $E$ & 25 & 0,58 \\
\hline Vitex megapotamica (Spreng.) Moldenke & tarumã & Lamiaceae & $\mathrm{N}$ & 25 & 0,58 \\
\hline Morus nigra L. & amoreira & Moraceae & $E$ & 22 & 0,51 \\
\hline Cinnamomum burmanni (Nees \& T. Nees) & falsa-canela & Lauraceae & $E$ & 21 & 0,49 \\
\hline
\end{tabular}

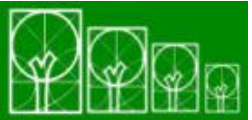

$\mathbf{S} \cdot \mathbf{B} \cdot \mathbf{A} \cdot \mathbf{U}$ Soc. Bras. de Arborização Urbana 


\begin{tabular}{|c|c|c|c|c|c|}
\hline Nome científico & Nome popular & Família & Ocorrência & FA & $\begin{array}{l}\mathrm{FR} \\
(\%)\end{array}$ \\
\hline \multicolumn{6}{|l|}{ Blume } \\
\hline Hibiscus rosa-sinensis L. & ibisco & Malvaceae & $E$ & 21 & 0,49 \\
\hline Hovenia dulcis Thunb. & uva-do-japão & Rhamnaceae & $E$ & 21 & 0,49 \\
\hline Spathodea nilotica Seem. & tulipeira & Bignoniaceae & $E$ & 21 & 0,49 \\
\hline Citrus sp. L. & limoeiro & Rutaceae & $E$ & 20 & 0,46 \\
\hline Syzygium cumini (L.) Skeels & jambolão & Myrtaceae & $E$ & 20 & 0,46 \\
\hline Chorisia speciosa A. St.-Hil. & paineira & Malvaceae & $E$ & 17 & 0,39 \\
\hline Delonix regia (Bojer ex Hook.) Raf. & flamboiant & Fabaceae & $E$ & 17 & 0,39 \\
\hline Eriobotrya japonica (Thunb.) Lindl. & nêspera & Rosaceae & $E$ & 16 & 0,37 \\
\hline Luehea divaricata Mart. & açoita-cavalo & Malvaceae & $\mathrm{N}$ & 16 & 0,37 \\
\hline Myrsine sp. L. & capororoca & Primulaceae & $\mathrm{N}$ & 16 & 0,37 \\
\hline Senna multijuga (Rich.) H.S. Irwin \& Barn. & pau-cigarra & Fabaceae & $E$ & 15 & 0,35 \\
\hline Bauhinia variegata $\mathrm{L}$. & pata-de-vaca & Fabaceae & $E$ & 14 & 0,32 \\
\hline $\begin{array}{l}\text { Enterolobium contortisiliquum (Vell.) } \\
\text { Morong }\end{array}$ & timbaúva & Fabaceae & $\mathrm{N}$ & 14 & 0,32 \\
\hline Eugenia involucrata DC. & cerejeira & Myrtaceae & $\mathrm{N}$ & 13 & 0,30 \\
\hline Nerium oleander L. & espirradeira & Apocynaceae & $E$ & 13 & 0,30 \\
\hline Roystonea oleracea (Jacq.) O.F. Cook & palmeira-imperial & Arecaceae & $E$ & 13 & 0,30 \\
\hline Schinus terebinthifolius Raddi & aroeira-vermelha & Anacardiaceae & $\mathrm{N}$ & 13 & 0,30 \\
\hline Schefflera actinophylla (Endl.) Harms & guarda-chuva & Araliaceae & $E$ & 12 & 0,28 \\
\hline Pinus sp. L. & pinus & Pinaceae & $E$ & 11 & 0,25 \\
\hline $\begin{array}{l}\text { Archontophoenix alexandrae } \\
\text { (F.Muell.)H.Wen.\& Dru. }\end{array}$ & palmeira-real & Arecaceae & $E$ & 10 & 0,23 \\
\hline Bauhinia forficata Link & pata-de-vaca & Fabaceae & $\mathrm{N}$ & 10 & 0,23 \\
\hline Inga vera Willd. & ingá-banana & Fabaceae & $\mathrm{N}$ & 9 & 0,21 \\
\hline $\begin{array}{l}\text { Senna macranthera (DC.exCollad.) } \\
\text { H.S.Irwin\&Barne. }\end{array}$ & fedegoso & Fabaceae & $E$ & 9 & 0,21 \\
\hline Callistemon sp. R. Br. & celistemone & Myrtaceae & $E$ & 8 & 0,19 \\
\hline Erythroxylum argentinum O.E. Schulz & cocão & Erythroxylaceae & $\mathrm{N}$ & 8 & 0,19 \\
\hline Inga marginata Willd. & ingá-feijão & Fabaceae & $\mathrm{N}$ & 8 & 0,19 \\
\hline Punica granatum $\mathrm{L}$. & romã & Lythraceae & $\mathrm{N}$ & 8 & 0,19 \\
\hline Thuja orientalis L. & tuia-da-china & Cupressaceae & $E$ & 8 & 0,19 \\
\hline Albizia sp.Durazz. & albizia & Fabaceae & $E$ & 7 & 0,16 \\
\hline Duranta repens L. & pingo-de-ouro & Verbenaceae & $E$ & 7 & 0,16 \\
\hline Salix babylonica L. & chorão & Salicaceae & $E$ & 7 & 0,16 \\
\hline Acer sp. L. & acer & Sapindaceae & $E$ & 5 & 0,12 \\
\hline Euphorbia cotinifolia L. & leiteiro-vermelho & Euphorbiaceae & $E$ & 5 & 0,12 \\
\hline Ocotea puberula (Rich.) Nees & canela & Lauraceae & $\mathrm{N}$ & 5 & 0,12 \\
\hline Pterocarya sp. Kunth & piterocaria & Juglandaceae & $E$ & 5 & 0,12 \\
\hline Terminalia catappa L. & amendoeira & Combretaceae & $E$ & 5 & 0,12 \\
\hline Cedrela fissilis Vell. & cedro & Meliaceae & $\mathrm{N}$ & 4 & 0,09 \\
\hline Grevillea robusta A. Cunn. ex R. Br. & carvalho-sodoso & Proteaceae & $E$ & 4 & 0,09 \\
\hline
\end{tabular}

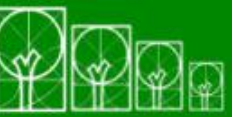

S · B · A $\cdot$ U Soc. Bras. de Arborização Urbana 


\begin{tabular}{|c|c|c|c|c|c|}
\hline Nome científico & Nome popular & Família & Ocorrência & FA & $\begin{array}{l}\mathrm{FR} \\
(\%) \\
\end{array}$ \\
\hline Tecoma stans (L.) Juss. ex Kunth & ipê-de-jardim & Bignoniaceae & $E$ & 4 & 0,09 \\
\hline Tibouchina granulosa (Desr.) Cogn. & quaresmeira & Melastomataceae & $E$ & 4 & 0,09 \\
\hline Acca sellowiana (O. Berg) Burret & goiaba-serrana & Myrtaceae & $\mathrm{N}$ & 3 & 0,07 \\
\hline Bougainvillea glabra Choisy & buganvile & Nyctaginaceae & $E$ & 3 & 0,07 \\
\hline Brunfelsia uniflora (Pohl) D. Don & primavera & Solanaceae & $\mathrm{N}$ & 3 & 0,07 \\
\hline Campomanesia rhombea O. Berg & guabiroba & Myrtaceae & $\mathrm{N}$ & 3 & 0,07 \\
\hline Eucalyptus sp. L'Hér. & eucalipto & Myrtaceae & $E$ & 3 & 0,07 \\
\hline Ficus microcarpa L. f. & $\begin{array}{l}\text { figueira-folha-miúda- } \\
\text { exótica }\end{array}$ & Moraceae & $E$ & 3 & 0,07 \\
\hline Mangifera indica L. & manga & Anacardiaceae & $E$ & 3 & 0,07 \\
\hline Murraya paniculata (L.) Jack & murraia & Rutaceae & $E$ & 3 & 0,07 \\
\hline Nectandra megapotamica (Spreng.) Mez & canela & Lauraceae & $\mathrm{N}$ & 3 & 0,07 \\
\hline Plumeria rubra L. & jasmim-manga & Apocynaceae & $E$ & 3 & 0,07 \\
\hline Quercus sp. L. & quercus & Fagaceae & $E$ & 3 & 0,07 \\
\hline Salix humboldtiana Willd. & salso-chorão & Salicaceae & $\mathrm{N}$ & 3 & 0,07 \\
\hline Schinus molle L. & aroeira-mansa & Anacardiaceae & $\mathrm{N}$ & 3 & 0,07 \\
\hline Tibouchina sellowiana Cogn. & quaresmeira & Melastomataceae & $\mathrm{N}$ & 3 & 0,07 \\
\hline Araucaria bidwillii Hook. & pinheiro-australiano & Araucariaceae & $E$ & 2 & 0,05 \\
\hline Araucaria columnaris Hook. & pinheiro-colunar & Araucariaceae & $E$ & 2 & 0,05 \\
\hline Carica papaya L. & mamão & Caricaceae & $E$ & 2 & 0,05 \\
\hline Caryota urens L. & rabo-de-peixe & Arecaceae & $E$ & 2 & 0,05 \\
\hline Cassia fistula $\mathrm{L}$. & chuva-de-ouro & Fabaceae & $E$ & 2 & 0,05 \\
\hline Cupressus sp. L. & cupressus & Cupressaceae & $E$ & 2 & 0,05 \\
\hline Euphorbia pulcherrima Willd. ex Klotzsch & bico-de-papagaio & Euphorbiaceae & $E$ & 2 & 0,05 \\
\hline Ficus cestrifolia Schott ex Spreng. & figueira-miúda & Moraceae & $\mathrm{N}$ & 2 & 0,05 \\
\hline Hibiscus mutabilis L. & rosa-louca & Malvaceae & $E$ & 2 & 0,05 \\
\hline Phoenix sp. L. & tamareira & Arecaceae & $E$ & 2 & 0,05 \\
\hline Piptocarpha angustifolia Dusén ex Malme & vassoura & Asteraceae & $\mathrm{N}$ & 2 & 0,05 \\
\hline Sesbania virgata (Cav.) Pers. & sesbânia & Fabaceae & $E$ & 2 & 0,05 \\
\hline Syzygium jambos (L.) Alston & jambo & Myrtaceae & $E$ & 2 & 0,05 \\
\hline Ateleia glazioviana Baill. & timbó & Fabaceae & $\mathrm{N}$ & 2 & 0,05 \\
\hline Zanthoxylum sp. L. & mamica-de-cadela & Rutaceae & $\mathrm{N}$ & 2 & 0,05 \\
\hline Acacia mearnsii De Wild. & acácia-negra & Fabaceae & $E$ & 1 & 0,02 \\
\hline Acacia podalyriifolia A. Cunn. ex G. Don & acácia- mimosa & Fabaceae & $E$ & 1 & 0,02 \\
\hline Araucaria angustifolia (Bertol.) Kuntze & pinheiro-brasileiro & Araucariaceae & $\mathrm{N}$ & 1 & 0,02 \\
\hline Artocarpus heterophyllus Lam. & jaca & Moraceae & $E$ & 1 & 0,02 \\
\hline Butia capitata (Mart.) Becc. & butiá & Arecaceae & $\mathrm{N}$ & 1 & 0,02 \\
\hline Camellia sp. L. & camélia & Theaceae & $E$ & 1 & 0,02 \\
\hline $\begin{array}{l}\text { Campomanesia guazumifolia (Cambess.) } \\
\text { O. Berg }\end{array}$ & guabiroba & Myrtaceae & $\mathrm{N}$ & 1 & 0,02 \\
\hline Cassia leptophylla Vogel & falso-barbatimão & Fabaceae & $E$ & 1 & 0,02 \\
\hline
\end{tabular}

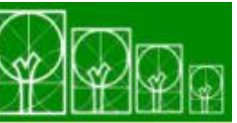




\begin{tabular}{|c|c|c|c|c|c|}
\hline Nome científico & Nome popular & Família & Ocorrência & FA & $\begin{array}{l}\mathrm{FR} \\
(\%) \\
\end{array}$ \\
\hline Casuarina equisetifolia L. & casuarina & Causuarinaceae & $E$ & 1 & 0,02 \\
\hline Cestrum corymbosum Schltdl. & coerana & Solanaceae & $E$ & 1 & 0,02 \\
\hline $\begin{array}{l}\text { Cordia americana (L.) Gottschling \& J.S. } \\
\text { Mill. }\end{array}$ & guajuvira & Boraginaceae & $\mathrm{N}$ & 1 & 0,02 \\
\hline Cupania vernalis Cambess. & camboatá-vermelho & Sapindaceae & $\mathrm{N}$ & 1 & 0,02 \\
\hline Eugenia brasiliensis Lam. & grumixama & Myrtaceae & $\mathrm{N}$ & 1 & 0,02 \\
\hline Euphorbia tirucalli L. & aveloz & Euphorbiaceae & $E$ & 1 & 0,02 \\
\hline Ficus elastica Roxb. ex Hornem. & falsa-seringueira & Moraceae & $E$ & 1 & 0,02 \\
\hline Ficus enormis (Mart. ex Miq.) Mart. & figueira-do-mato & Moraceae & $\mathrm{N}$ & 1 & 0,02 \\
\hline Machaerium stiptatum. Pers. & sapuva & Fabaceae & $\mathrm{N}$ & 1 & 0,02 \\
\hline Malvaviscus arboreus Cav. & malvavisco & Malvaceae & $E$ & 1 & 0,02 \\
\hline Mimosa bimucronata (DC.) Kuntze & maricá & Fabaceae & $\mathrm{N}$ & 1 & 0,02 \\
\hline Myrciaria tenella (DC.) O. Berg & guamirim & Myrtaceae & $\mathrm{N}$ & 1 & 0,02 \\
\hline Parkinsonia aculeata L. & cina-cina & Fabaceae & $\mathrm{N}$ & 1 & 0,02 \\
\hline Pleomele reflexa (Lam.) N.E. Br. & dracena-malaia & Liliaceae & $E$ & 1 & 0,02 \\
\hline Plinia trunciflora (O. Berg) Kausel & jaboticaba & Myrtaceae & $\mathrm{N}$ & 1 & 0,02 \\
\hline Podocarpus lambertii Klotzsch ex Endl. & podocarpus & Podocarpaceae & $\mathrm{N}$ & 1 & 0,02 \\
\hline Sapium glandulosum (L.) Morong & pau-leiteiro & Euphorbiaceae & $\mathrm{N}$ & 1 & 0,02 \\
\hline Schinus lentiscifolius Marchand & aroeira & Anacardiaceae & $\mathrm{N}$ & 1 & 0,02 \\
\hline Total & & & & 4318 & 100 \\
\hline
\end{tabular}

A arborização apresentou-se constituída de indivíduos com alturas nos intervalos entre 2 a 5m; 5 a $10 \mathrm{~m}$ e 10 a $20 \mathrm{~m}$, sendo $34,8 \%, 44,2 \%$ e $21 \%$, respectivamente, demonstrando o predomínio de árvores de médio e grande porte. A maioria dos indivíduos $(48,7 \%)$ apresentou classes de diâmetro de copa entre 0 a $5 \mathrm{~m}$ e apenas $13,1 \%$ dos exemplares apresentaram diâmetro maior ou igual a $10 \mathrm{~m}$. Acredita-se que apesar da maior frequência de indivíduos de maior altura, estes apresentam suas copas com menor diâmetro devido à poda de manutenção regular efetuada principalmente nas árvores em conflito com a rede elétrica.

Árvores muito altas, de copas frondosas e raízes proeminentes são conflitantes com toda a estrutura urbana, como rede elétrica, redes de esgoto e pluvial, calçamentos e construções (GREY; DENEKE,1978). Estas sofrem contínua manutenção através da prática de poda drástica tanto na copa como nas raízes, facilitando a infestação por parasitas como ervas-de-passarinho (gêneros Phoradendron e Phrygilanthus) (AGUIAR; SOARES; MARTAU, 1979) e doenças desencadeadas por patógenos, levando ao risco de queda do indivíduo. 
A espécie de maior frequência, Jacaranda mimosifolia, apresenta grande porte, copa frondosa e de sistema radicular profundo. Este indivíduo encontra-se amplamente distribuído sob rede elétrica, fazendo com que seja realizada poda periódica, desestabilizando o vegetal, ocasionando alto índice de tombamentos e queda de galhos, sendo responsável por $13 \%$ da remoção de árvores do município de Porto Alegre (SMAM, 2002).

Apesar das intervenções feitas na arborização, os resultados do estado fitossanitário demonstraram que $72,9 \%$ dos indivíduos estão em bom estado, enquanto que em $20 \%$ e $6,8 \%$ dos exemplares a fitossanidade é regular e ruim, respectivamente, demonstrando a boa saúde da vegetação, pois poucos indivíduos encontram-se em estado degenerativo nos passeios públicos diagnosticados. A qualidade dos vegetais é de suma importância na conservação do equilíbrio ecológico (PEREIRA et al., 2005), assim a manutenção da qualidade das árvores propicia habitats saudáveis para o aparecimento de outras espécies da flora e fauna, fazendo com que as interações ecológicas sejam maiores, conferindo a harmonia da vegetação com a urbanização (UTHKHEDE et al., 1997).

Entre as 122 espécies amostradas, 77 são de origem exótica da flora do Rio Grande do Sul, representando $63 \%$ do total, enquanto apenas 45 espécies são nativas. Jacaranda mimosifolia e Handroanthus avellanedae foram as espécies mais frequentes neste estudo, sendo as mesmas de origem exótica da flora da região. Estes resultados demostram que apesar de Porto Alegre ser uma das cidades mais arborizadas do Brasil, grande parte das espécies que compõem a arborização são exóticas. No que se refere à ocorrência das espécies registradas, a maior parte tem sua origem fora do continente americano. Este é o caso de várias espécies muito apreciadas no paisagismo no Brasil, como Ligustrum lucidum, Ficus benjamina, Lagerstroemia indica, Hibiscus rosa-sinensis, Melia azedarach, Hovenia dulcis, Nerium oleander, Delonix regia, Spathodea campanulata e Grevillea robusta.

Ficus benjamina gera muitos problemas na arborização como o tombamento de outras árvores, rachaduras de calçamento e construções. Esta figueira exótica apresenta crescimento rápido e raízes que podem causar inúmeros danos (SMAM, 2002). A espécie africana Spathodea campanulata é usada amplamente na arborização e no paisagismo por apresentar características ornamentais. No entanto, foi observado por Trigo e Santos (2000) que suas flores apresentam alcalóides tóxicos que podem matar abelhas e beija-flores nativos. Lagerstroemia indica, a extremosa, aparece em terceiro lugar entre as espécies mais frequentes na arborização dos bairros avaliados. Segundo Biondi e Althaus (2005),

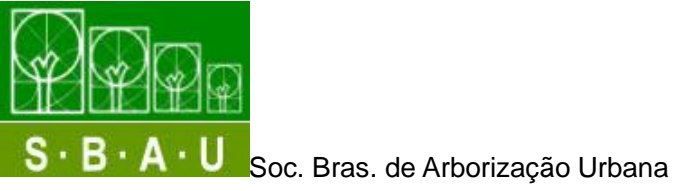


esta pode ser frequentemente atacada por míldio, mancha foliar, mancha negra e podridão radicular, comprometendo seu estado fitossanitário e servindo como vetor de pragas para indivíduos próximos além da suscetibilidade a infestação por erva-de-passarinho.

Também foram encontradas espécies exóticas invasoras como o Pinus sp. e Hovenia dulcis; ambas relatada como extremamente invasoras em diversos ambientes florestais e campestres no sul do Brasil (INSTITUTO HÓRUS, 2011). O Pinus sp. pode acarretar mudanças significativas na estrutura da vegetação e modificar o ciclo de nutrientes. Sua síndrome de dispersão, anemocórica, propicia vasta disseminação, sendo elemento que pode estabelecer populações autorregenerativas e expandir-se sobre áreas de campos naturais (ZALBA; VILLAMIL, 2002). Já Hovenia dulcis tem se mostrado invasora em áreas de Florestas Ombrófilas e Estacionais (INSTITUTO HÓRUS, 2011).

Umas das espécies mais abundantes, o ligustro (Ligustrum lucidum), é muito comum nas cidades Sul-Brasileiras (BACKES; IRGANG, 2004). No Paraná é considerada invasora na Floresta Ombrófila Mista (INSTITUTO HÓRUS, 2011). Os frutos do ligustro, se consumidos, são tóxicos para os humanos podendo causar reações adversas (INSTITUTO HÓRUS, 2011) e os pólens das flores desencadearem alergias (BACKES; IRGANG, 2004; BIONDI; ALTHAUS, 2005).

Inventários realizados em outras cidades brasileiras demonstram o predomínio das espécies exóticas sobre as nativas (neste caso, considerando as nativas do Brasil como um todo). Em Campina Grande - PB, Dantas e Souza (2004) registraram frequências de 67,2\% para árvores exóticas e 32,8\% para nativas do Brasil. Em Lageado - RS, Ruschel e Leite (2002) encontraram valores mais equilibrados: $53,6 \%$ de exóticas e $46,4 \%$ de nativas. $E$ em Pato Branco - PR, Silva et al. (2007) registraram valores muito próximos aos de Lageado: $53,2 \%$ de exóticas e $46,8 \%$ de nativas.

De acordo com a Resolução COMAM no 05/2006 que dispõe sobre o Plano Diretor de Arborização Urbana de Porto Alegre (SMAM, 2002), no Art. 7ํㅡ, no qual trata da melhoria da qualidade de vida e equilíbrio ambiental, observa-se que se deve utilizar predominantemente espécies nativas regionais em projetos de arborização de ruas, avenidas e de terrenos privados, respeitando o percentual mínimo de $70 \%$ de espécies nativas. No entanto, conforme os resultados encontrados no presente inventário nota-se que esta premissa não está sendo regularmente implantada. Além disso, a resolução propõe diversificar as espécies utilizadas na arborização pública e privada como forma de assegurar a estabilidade e a preservação da floresta urbana.

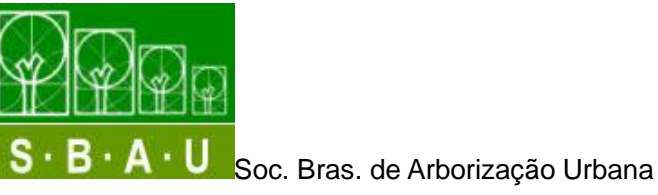


Assim, considera-se importante a realização de um plano efetivo de monitoramento e manutenção com base no Plano Diretor de Arborização existente para o município. É necessário avaliar e projetar o uso de nativas adequando a estrutura urbana e o local de plantio a cada tipo de espécie, sempre respeitada a diversidade biológica que confere integridade ao patrimônio natural, essencial à qualidade de vida.

\section{CONCLUSÃO}

Conclui-se que embora as árvores nas vias públicas de Porto Alegre sejam abundantes e um exemplo para outros grandes centros urbanos, a grande frequência de espécies exóticas foi diagnosticada e deve ser evitada, principalmente quanto às potencialmente invasoras. Sugere-se a substituição gradativa dos indivíduos exóticos já existentes por nativos que apresentem características ecológicas compatíveis com meio urbano. São sugestões viáveis a realização de novos plantios, substituições e manutenções que levem em conta as condições adequadas para que a vegetação contribua positivamente com os objetivos da arborização nos centros urbanos.

\section{REFERÊNCIAS BIBLIOGRÁFICAS}

AGUIAR, L. W., SOARES, Z. F., MARTAU, L. Nota sobre Phrygilanthus acutifolius (R. \& PAV.) EICHL. e Phoradendron martianum TREL. nos Parques Farroupilha e Paulo Gama, Porto Alegre, RS, BRASIL. Iheringia Sér. Bot., Porto Alegre, v. 24, p. 83-89, 1979.

ANDRADE, T. O. Inventário e Análise da Arborização Viária da Estância Turística de Campos do Jordão, SP. Piracicaba, São Paulo, 2002. Dissertação (Mestrado) - Escola Superior de Agricultura, Universidade de São Paulo.

APG - ANGIOSPERM PHYLOGENY GROUP. An update of the Angiosperm Phylogeny Group classification for the orders and families of flowering plants: APG III. Botanical Journal of the Linnean Society, Londres, v. 161, p. 105-121, 2009. 
BACKES, P.; IRGANG, B. Mata Atlântica: as árvores e a paisagem. Porto Alegre: Paisagem do Sul, 2004.

BIONDI, D.; ALTHAUS, M. Árvores de rua de Curitiba: cultivo e manejo. Curitiba: FUPEF, 2005.

DANTAS, I. C.; SOUZA, C. M. C. Arborização urbana na cidade de Campina Grande - PB: Inventário e suas espécies. Revista de Biologia e Ciências da Terra. Campina Grande, v. 4, n. 2, dez. 2004.

FARIA, J. L. G.; MONTEIRO, E. A.; FISCH, S. T. Arborização de vias públicas do município de Jacareí - SP. Revista da Sociedade Brasileira de Arborização Urbana. Piracicaba, v. 2, n. 4, p. 20-33, dez. 2007.

FILHO, D. F. S.; PIZETTA, P. U. C.; ALMEIDA, J. B. S. A.; PIZETTA, K. F. L.; FERRAUDO, A. S. Banco de dados relacional para cadastro, avaliação e manejo da arborização em vias públicas. Revista Árvore, v. 26, n. 5, p. 629-642, 2002.

GODOY, A. L. P. Cidade e Meio Ambiente: O planejamento da arborização de Pirassununga/SP. Rio Claro: IGCE/UNESP, 1995. Dissertação (Mestrado) - Universidade Estadual Paulista "Júlio de Mesquita Filho", campus de Rio Claro.

GREY, G.; DENEKE, F. Urban forestry. New York: John Wiley, 1978.

INSTITUTO HÓRUS. Exóticas invasoras: Fichas técnicas. 2011. Disponível em: < http:// www.Institutohorus.org.br/inf._fichas.htm. >. Acesso em 04/05/2011.

JACINTO, J. M. M. Análise silvicultural urbana de seis espécies florestais utilizadas na arborização de Brasília. Brasília, 2001. Dissertação (Mestrado) - Departamento de Engenharia Florestal, Faculdade de Tecnologia, Universidade de Brasília.

LEITE, P. F.; KLEIN, R. M. Vegetação. Levantamento de recursos naturais. Rio de Janeiro: IBGE, 1990.

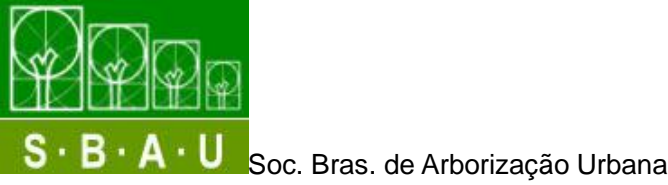

REVSBAU, Piracicaba - SP, v.6, n.3, p.189-206, 2011 
LIMA, A. M. L. P. Árvores de rua. Revista Globo Ciência. São Paulo, № 44, 1995.

LORENZI, H. Árvores Brasileiras: manual de identificação e cultivo de plantas arbóreas do Brasil. São Paulo: Instituto Plantarum, 2002a.

Árvores Brasileiras: manual de identificação e cultivo de plantas arbóreas do Brasil. São Paulo: Instituto Plantarum, 2002b.

LORENZI, H.; SOUZA, H. M; TORRES, M. A. V.; BACHER, L. B. Árvores exóticas no Brasil: madeiras, ornamentais e aromáticas. São Paulo: Instituto Plantarum, 2003.

MASCARÓ, L.; MASCARÓ, J. Vegetação urbana. Porto Alegre: Editora da URRS, 2002.

MILANO, M. S. Avaliação e análise da arborização de ruas de Curitiba - PR. Dissertação de Mestrado. Curitiba: Curso de pós-graduação em Engenharia Florestal - UFPR, 1984.

MILANO, M. S.; DALCIN, E. C. Arborização de vias públicas. Rio de Janeiro: Light, 2000.

PALERMO, A. Arborização. São Paulo: CESP, 1985.

PEREIRA, G. A.; MONTEIRO, C. S.; CAMPELO, M. A.; MEDEIROS, C. O uso de espécies vegetais, como instrumento de biodiversidade da avifauna silvestre, na arborização pública: o caso do Recife. Atualidades Ornitológicas. n. 125, p. 1-15, 2005.

RUSCHEL, D.; LEITE, S .L. C. Arborização Urbana em uma Área da Cidade de Lajeado, Rio Grande do Sul, Brasil. Caderno de Pesquisa Sér. Bio. Santa Cruz do Sul, v. 14, n. 1, p. 724, jun. 2002.

SANCHOTENE, M. C. C. Situação das Áreas Verdes e da Arborização Urbana em Porto Alegre. In: $1^{\circ}$ Encontro Brasileiro Sobre Arborização Urbana. Contribuições técnicas científicas. Curitiba, FUPEF, 1990. p. 35. 
SANTAMOUR JÚNIOR, F. S. Trees for urban planting: diversity uniformity, and common sense. Agriculture Research Service. Washington: U. S. National Arboretum, 2002.

SANTOS, N. R. Z.; TEIXEIRA, I. F. Arborização de vias públicas: ambiente x vegetação. Rio Grande do Sul: Instituto Souza Cruz, 2001.

SECRETARIA MUNICIPAL DO MEIO AMBIENTE (SMAM). Plano Diretor de Arborização de Porto Alegre. Cartilha da Arborização Urbana. Porto Alegre, 2002.

SILVA, L. M.; HASSE, I.; MOCCELIN, R.; ZBORALSKI, A. R. Arborização de vias públicas e a utilização de espécies exóticas: o caso do bairro Centro de Pato Branco/PR. Scientia Agraria. Paraná, v. 8, n. 1, p. 47-53, 2007.

SOBRAL, M.; JARENKOW, J. A.; BRACK, P.; IRGANG, B. E.; LAROCCA, J.; Rodrigues, R. S. Flora Arbórea e Arborescente do Rio Grande do Sul, Brasil. São Carlos: RiMa Editora, 2006.

SOUZA, V. C.; LORENZI, H. Botânica Sistemática: Guia ilustrado para identificação das famílias de Angiospermas da flora brasileira, baseado em APG II. São Paulo: Instituto Plantarum, 2008.

TEIXEIRA, M. B.; COURA NETO, A. B.; PASTORE, U.; RANGEL FILHO, A. L. R. Levantamento de recursos naturais. Rio de Janeiro: IBGE, 1986.

TOLEDO, D. V.; PARENTE, P. R. Arborização urbana com essências nativas. Boletim Técnico do Instituto Florestal. São Paulo, v. 42, mai, 1988.

TRIGO, J. R.; SANTOS, W. F. Insect mortality in Spathodea campanulata Beauv. (Bignoniaceae) flowers. Revista Brasileira de Biologia. São Carlos, v. 60, n. 3, p. 537-538, 2000. 
UTHKHEDE, R.; STEPHEN, B; WONG, S. Control of Phytophthora lateralis root rot of Lawson Cypress with Enterobacter aerogenes. Journal of Arboriculture. v. 23, n. 4, p. 144146, 1997.

VELASCO, G. Del N.; LIMA, A. M. L. P.; COUTO, H. T. Z. Análise comparativa dos custos de diferentes redes de distribuição de energia elétrica no contexto da arborização urbana. Revista Árvore. Viçosa, v. 30, n. 4, p. 679-686, jul./ago. 2006.

ZALBA, S. M.; VILLAMIL, C. B. Woody Plant Invasion in Relictual Grasslands. Biological Invasions. EUA, v. 4, n. 1-2, p. 55-72, 2002.

ZILLER, S. R. Os processos de degradação ambiental originados por plantas invasoras. Revista Ciência Hoje. Rio de Janeiro, n.178, dez. 2001. 